\title{
Study of 41 Cases of Pregnancy after Uterine Leiomyoma Treatment by Uterine Artery Embolization (UAE)
}

\author{
Takahisa Kojima1, Hidefumi Fujisawa², Kumiko Koyama² \\ ${ }^{1}$ Amor Clinic, Yokohama, Japan \\ ${ }^{2}$ Department of Radiology, Showa University Northern Yokohama Hospital, Yokohama, Japan \\ Email: masasugimoto74@gmail.com
}

How to cite this paper: Kojima, T., Fujisawa, H. and Koyama, K. (2018) Study of 41 Cases of Pregnancy after Uterine Leiomyoma Treatment by Uterine Artery Embolization (UAE). Open Journal of Obstetrics and Gynecology, 8, 1255-1259.

https://doi.org/10.4236/ojog.2018.813127

Received: July 2, 2018

Accepted: October 27, 2018

Published: October 30, 2018

Copyright $\odot 2018$ by authors and Scientific Research Publishing Inc. This work is licensed under the Creative Commons Attribution International License (CC BY 4.0).

http://creativecommons.org/licenses/by/4.0/

\begin{abstract}
Uterine leiomyoma, a benign tumor, may be treated with hormone therapy, conventional surgical resection and uterine artery embolization (UAE): this paper reports the study on safety after UAE treatment. Pregnancy processes and prognoses of a total of 41 cases from 39 patients (two women were pregnant twice) who succeeded to pregnancy after UAE treatment in our clinic were studied. The average age of patients was 34.5 (29 - 40). Patients included 24 cases of multiple uterine leiomyoma and 14 cases of solitary leiomyoma; the average size of leiomyoma was $388.3 \mathrm{~cm}^{3}\left(15-1059 \mathrm{~cm}^{3}\right)$ and the average period between UAE operation and pregnancy was 26.5 months $(1-120)$. Causes of pregnancy were 29 cases of natural pregnancy and 12 cases of infertility treatment: 28 cases succeeded in delivery, 7 cases resulted in spontaneous abortion, and 6 cases are ongoing. Premature birth, placental abnormality and low-weight babies were observed in 3, 3, and 1 cases, respectively; the latter 1 case was of triplets. Although the incidence of placental abnormality was a little high, no other serious complications were observed, suggesting that pregnancy after UAE has no problem from the obstetric point of view. In the future, UAE is considered to be worthwhile if carefully done for cases who want to become pregnant.
\end{abstract}

\section{Keywords}

Embolization, Gelatin Sponge Particles, Leiomyoma, Pumping Method, Uterine Artery Embolization (UAE), Uterine Leiomyoma, Pregnancy, Risk

\section{Introduction}

Other than medical and surgical therapies, a method to treat leiomyoma by em- 
bolizing the feeding artery was reported in 1995, in which embolizing material is injected through a catheter inserted into the uterine artery [1].

This uterine artery embolization (UAE) has gradually been accepted as an option to treat uterine leiomyoma. The following points are raised as advantages of UAE: at first preservation of the uterus, followed by a reduced physical and economic burden, and absence of surgical blood loss, intraperitoneal adhesion and postoperative scarring, etc. Reported disadvantages are: difficulty to manage postoperative pain, rare occurrence of decreased ovarian function, difficulty to be pregnant and occurrence of many obstetric complications. In Japan, surgical therapy is selected overwhelmingly to treat uterine leiomyoma. However, most patients who visit our clinic eagerly want to keep the uterus or do not want surgery itself after they know the existence of UAE via the social network service.

This paper assessed the risk for pregnancy after treatment of UAE.

\section{Materials and Methods}

The clinic has introduced UAE by using gelatin sponge particles prepared by the pumping method [2] from 2001 to 2015 and has done UAE in the outpatient department without requiring hospitalization. A total of 1200 patients received UAE treatment, among which 240 patients wished to have babies, and 39 patients became pregnant (41 cases, two women became pregnant twice). UAE was done after obtaining enough "understanding and consent" from patients who wish for UAE on the understanding that as a treatment of uterine leiomyoma they can choose a treatment among the methods of surgical removal, UAE, hormonal therapy, etc. Under anesthesia of UAE, a catheter was inserted from the right femoral artery and a catheter was placed in bilateral uterine arteries. UAE was done according to the method reported previously by us [2].

Briefly, uterine arteries were inspected to confirm their positions and then a semi-liquid gelatin sponge as an embolic material (made by the pumping method) was infused into the bilateral uterine artery to embolize the arteries. The infusion of the embolic material was stopped when the horizontal branch or ascending branch of the uterine artery was embolized. Vital check and pain managements were intensively done for 24 hours after UAE. For patients with pain, the painkiller Tramadol was usually sustainably administered subcutaneously and the patients were allowed to return home after 24 hours. At 1 week, 1 month, and 6 months after the operation, both ultrasonic examination and magnetic resonance imaging (MRI) examinations were done. When patients became pregnant after UAE, the doctors for all of the patients reported us about the progress of pregnancy and the results of delivery: the possibility might have existed that some pregnant patients might not have told the doctors.

\section{Results}

Among a total of 1200 UAE cases performed during 15 years, 1166 cases (97.1\%) were effective, 34 cases were ineffective $(2.8 \%) ; 2$ other cases were unable to be 
performed. Among these cases, 39 cases resulted in pregnancy, and the number of pregnancies was 41 pregnancies, including two women who were pregnant twice.

Age of each pregnancy case at the time of UAE was $34.5 \pm 3.2$ years in average ( 29 to 40 years old). The susceptible age of uterine leiomyoma is said to be age 30 or more; in this study the youngest case was 29 years old and the oldest age was 40 years old.

- Number of uterine leiomyomas: 24 multiple cases (63.8\%), 14 single cases (36.2\%), 3 giant cases (8.3\%). Multiple cases tended to be slightly more frequent with only 3 cases of gigantic fibroids that developed beyond the umbilical region.

- Size of uterine leiomyoma: average $388.3 \pm 283.8 \mathrm{~cm}^{3}\left(15-1059 \mathrm{~cm}^{3}\right)$.

- Major uterine leiomyoma sites: 0 case was subserous, 18 cases were intramuscular, 18 cases were submucosal, 3 cases were cervical.

- Reduction rate of uterine leiomyomas one year after UAE: average reduction rate was $59.2 \pm 21.4 \%(95.0 \%$ - 0\%) In 2 cases the uterine leiomyoma disappeared after UAE.

- The term between UAE and pregnancy: average $26.5 \pm 29.9$ months (1 month - 120 months). In 9 cases, patients became pregnant within 6 months after UAE.

- Natural pregnancy was 29 cases (70.9\%) and pregnancy after infertility treatment was 12 cases among which 9 cases were pregnancy by assisted productive technology.

- Among 41 cases of pregnancy were 7 spontaneous abortions (17.1\%), 18 normal deliveries (43.9\%), 10 caesarean sections (24.4\%) and 6 cases (14.6\%) of ongoing or unknown pregnancies.

- Average weight of babies at delivery: $2945 \mathrm{~g} \pm 592 \mathrm{~g}$ (3885 g - $1054 \mathrm{~g}: 3$ triplets).

- Obstetric complications were 3 cases of premature birth (29 weeks, 32 weeks, 33 weeks each), 2 cases of placenta previa, 1 case of adherent placenta, and 3 cases of placental abnormality which were slightly high. In one case of severe adherent placenta, the uterus was eventually removed due to abundant bleeding.

\section{Discussion}

Both merits and demerits exist for various treatments of uterine leiomyoma, such as surgical excision, UAE embolizing the uterine artery and focused ultrasound surgery that burns leiomyoma by ultrasound guided by MRI, etc. Many reports [3]-[10] show that UAE has almost the same therapeutic effect as surgical therapy. Both negative and positive opinions exist as to whether to do UAE for patients who hope for pregnancy in the future .Recently, the opinion that UAE is acceptable has increased from results that UAE is comparable to results of laparotomy [3] [4]. Surgical uterine excision results in problems, such as a high rate 
of recurrence of uterine leiomyoma accompanied by complications, including adhesion or blood loss. Pregnancy within half a year after nuclear excision is usually recommended because of recurrence. UAE was avoided for patients with huge leiomyoma and those whose ovary function was weakened.

Whether to apply UAE for patients who hope for babies has been controversial in the past. Many pregnancy cases after UAE have been reported [3]-[10]. The concerns are that UAE itself causes damage to the ovary and uterus leading to difficulty in pregnancy, and that even if a successful pregnancy is established, an obstetric complication is feared to increase later. After a UAE operation, the reported frequency of ovarian dysfunction is $2 \%-6 \%$, and in the clinic we had similar results: among a total of 1200 cases ovarian dysfunction after UAE was in 25 cases $(2.1 \%)$, among which most cases had a high follicle stimulating hormone value and a low anti-Mullerian hormone value before surgery. Therefore, a preliminary diagnosis may be possible for prediction to some extent before an UAE operation.

Regarding obstetric complications, one study [7] reported that UAE is inappropriate for patients who hope for a baby because of many obstetric complications that are reported, but many other studies accept UAE on the whole. For instance, Pisco et al. [4] reported the following results. They evaluated the outcome of pregnancy after an UAE operation for 74 patients who wanted to become pregnant. Of these 74 women, 44 of them became pregnant (59.5\%) of which five $(11.3 \%)$ of them had ongoing pregnancies and $39(88.7 \%)$ of them had finished pregnancies. Of the 39 pregnancies that had finished, 33 pregnancies were successful live births (84.6\%), four pregnancies ended in spontaneous abortions (10.3\%), one pregnancy ended in an induced abortion, and one pregnancy ended as stillbirth. As the successful live births are included 22 cesarean deliveries (66.6\%), two preterm deliveries at 36 weeks $(6.1 \%)$ and five deliveries of low birth weight. According to these results the Pisco study concluded that pregnancy after UAE appears to be safe.

Usually, placental anomalies tend to be slightly more frequent in patients receiving UAE treatment. In our study, 3 cases of placental abnormalities were observed in 28 cases of parturition: 2 cases were preplacental and 1 case was adhesion placenta. In the former cases, babies were safely delivered by caesarean section, but in the latter case of severe adhesion placenta, total hysterectomy could not be avoided.

To summarize, among 1200 patients who received UAE treatment, 241 (20.0\%) patients hoped to have babies, and 39 patients had 41 pregnancies (two women were pregnant twice). Among the pregnant patients, 28 cases were natural pregnancy and 13 cases became pregnant after infertility treatment. Normal delivery was in 18 cases, cesarean delivery was in 10 cases, spontaneous abortion was in 7 cases and 6 cases were ongoing. Of the above mentioned 28 pregnant patients, placental abnormalities were $3(10.7 \%)$, premature births were $3(10.7 \%)$ and low weight infants were only 1 case of triplets. 


\section{Conclusion}

In the future, UAE seems to be worthwhile to be carefully implemented in patient cases who wish to be pregnant.

\section{Conflicts of Interest}

The authors declare no conflicts of interest regarding the publication of this paper.

\section{References}

[1] Ravina, J.H., Herbreteau, D., Ciraru-Vigneron, N., Bouret, J.M., Houdart, E., Aymard, A., et al. (1995) Arterial Embolisation to Treat Uterine Myomata. Lancet, 346, 671-672. https://doi.org/10.1016/S0140-6736(95)92282-2

[2] Kojima, T., Taki, Y., Fujisawa, H. and Koyama, K. (2012) Leiomyoma Treatment by Uterine Artery Embolization Using Gelatin Sponge Prepared by the Pumping Method. Experimental and Therapeutic Medicine, 4, 781-784. https://doi.org/10.3892/etm.2012.688

[3] Firouznia, K., Ghanaati, H., Sanaati, M., Jalali, A.H. and Shakiba, M. (2009) Pregnancy after Uterine Artery Embolization for Symptomatic Fibroids: A Series of 15 Pregnancies. American Journal of Roentgenology, 192, 1588-1592. https://doi.org/10.2214/AJR.07.3904

[4] Pisco, J.M., Duarte, M., Bilhim, T., Cirurgiao, F. and Oliveira, A.G. (2011) Pregnancy after Uterine Fibroid Embolization. Fertility and Sterility, 95, 1121.e5-1121.8.

[5] Descargues, G., Mauger Tinlot, F., Douvrin, F., Clavier, E., Lemoine, J.P. and Marpeau, L. (2004) Menses Fertility and Pregnancy after Arterial Embolization for the Control of Postpartum Haemorrhage. Human Reproduction, 19, 339-343.

https://doi.org/10.1093/humrep/deh082

[6] Poggi, S.H., Yaeger, A., Wahdan, Y. and Ghidini, A. (2015) Outcome of Pregnancies after Pelvic Artery Embolization for Postpartum Hemorrhage: Retrospective Cohort Study. American Journal of Obstetrics \& Gynecology, 213, 576.e1-576.e5. https://doi.org/10.1016/j.ajog.2015.06.063

[7] Pron, G., Mocarski, E., Bennett, J., Vilos, G., Common, A., Vanderburgh, L., et al. (2005) Pregnancy after Uterine Artery Embolization for Leiomyomata: The Ontario Multicenter Trial. Obstetrics \& Gynecology, 105, 67-76. https://doi.org/10.1097/01.AOG.0000149156.07061.1f

[8] Rebarber, A., Fox, N.S., Eckstein, D.A., Lookstein, R.A. and Saltzman, D.H. (2009) Successful Bilateral Uterine Artery Embolization during an Ongoing Pregnancy. Obstetrics \& Gynecology, 113, 554-556. https://doi.org/10.1097/AOG.0b013e318193bfdf

[9] Soeda, S., Kyozuka, H., Suzuki, S., Yasuda, S., Nomura, Y. and Fujimori, K. (2014) Uterine artery Embolization for Uterine Arteriovenous Malformation Is Associated with Placental Abnormalities in the Subsequent Pregnancy: Two Cases Report. Fukushima Journal of Medical Science, 60, 86-90. https://doi.org/10.5387/fms.2013-13

[10] Walker, W.J. and McDowell, S.J. (2006) Pregnancy after Uterine Artery Embolization for Leiomyomata: A Series of 56 Completed Pregnancies. American Journal of Obstetrics \& Gynecology, 195, 1266-1271. https://doi.org/10.1016/j.ajog.2006.04.011 